Carla C.P. Verstappen

Bastiaan R. Bloem

\section{Too hot to handle: heat stroke in multiple system atrophy}

Received: 22 February 2006

Accepted: 27 April 2006

Published online: 3 April 2007

Sirs: Autonomic dysfunction is a common feature of multiple system atrophy (MSA). It can be incapacitating for patients, leading to urinary urge, severe constipation, altered sweating, and orthostatic hypotension with falls due to syncope [1]. Here, we present an unusual complication of autonomic dysfunction in a patient with definite MSA that came to light during the unusual hot summer of 2003 in the Netherlands.

A sixty-six year old man with a four year history of bladder hypocontractility, and a one year history of orthostatic hypotension, was referred to our Department of Neurology for further evaluation. The patient's main subjective complaint was lightheadedness

C.C.P. Verstappen

B.R. Bloem, MD, PhD

Dept. of Neurology

Parkinson Centre Nijmegen

Radboud University Nijmegen Medical

Centre

Nijmegen, The Netherlands

B.R. Bloem, MD, PhD ( $\square)$

Dept. of Neurology (935)

Parkinson Centre Nijmegen,

Radboud University Nijmegen Medical

Centre

P.O. Box 9101

6500 HB Nijmegen

The Netherlands

Tel.: +31-24/3613396

Fax: +31-24/3541122

E-Mail: b.bloem@neuro.umcn.nl while walking the stairs. He did not report muscle stiffness or slowness when walking. His upright posture had become slightly stooped during the preceding year. He further complained of a dry mouth, being unable to sweat, impotence, and problems with swallowing.

When first seen by us in March 2002, neurological examination revealed marked orthostatic hypotension (supine: 150/90 $\mathrm{mm} \mathrm{Hg}$; erect 3 minutes after rising: $90 / 70 \mathrm{~mm} \mathrm{Hg}$ ), and slight hypomimia of the face, but no bradykinesia or hypokinesia of the extremities. His voice was soft and monotone. Posture was slightly stooped, but gait was normal. The arms displayed subtle and symmetrical signs of rigidity, with cogwheeling. There was no resting tremor. The retropulsion test showed mild postural instability with a few steps retropulsion, but unaided recovery. Vibration sense in the lower legs was absent, with a positive Romberg sign. Tendon reflexes were symmetrically brisk in both the arms and legs, with Babinski responses in both feet. The patient was diagnosed as having a probable MSA of the striatonigral subtype. In the following year, he developed a marked, symmetrical bradykinesia and rigidity. Levodopa therapy was started and titrated to a maximal dose of 750 $\mathrm{mg} /$ day, with an only minimal improvement in his motor performance. He also received hydrocortisone to treat the orthostatic hypotension.

Magnetic resonance imaging of the brain showed slight generalized atrophy. [ ${ }^{123}$ I]IBZMSPECT showed bilaterally diminished striatal uptake, consisting with dopamine D2-receptor loss. Neurophysiological examination showed signs of a minor sensorimotor axonal polyneuropathy.

During an unusually hot summer period (by Dutch standards) in 2003, with temperatures of 30 degrees Celsius, the patient was admitted to our emergency room because he had been unconsciousness for four hours. General physical examination revealed a body temperature of 41 degrees Celsius, but the patient was not sweating anywhere. Neurological examination revealed somnolence, with a Glasgow Coma Score of Eyes $=4$, motor response $=6$ and verbal response $=2$. There were no signs of meningism or focal neurological deficits. Maligant neuroleptic syndrome was deemed very unlikely on clinical grounds, because there were no signs of extreme stiffness (rigidity was comparable to what we scored during an outpatient clinic visit 3 months earlier). Moreover, the patient was not receiving any neuroleptics, and at that time of the event levodopa therapy had not yet been initiated, so acute levodopa withdrawal or treatment failure as possible cause of malignant neuroleptic syndrome could be excluded.

Laboratory examination showed no abnormalities, including a normal leukocyte count $\left(9.1 \times 10^{9} / \mathrm{l}\right)$ and C-reactive protein $(7 \mathrm{mg} / \mathrm{l})$. A lumbar puncture was performed with a normal opening pressure $\left(18 \mathrm{~cm} \mathrm{H}_{2} \mathrm{O}\right)$, no leukocytes, and a normal protein and glucose content. Direct Gram staining of the cerebral spinal fluid showed no microorganisms.

Radiographs of the chest showed no infiltrates, a urinary culture demonstrated no bacteria, and blood cultures were negative. After active cooling with external cool pads, the body temperature rapidly decreased, and the patient quickly recovered. 
Two months later motor performance further declined, and levo-dopa therapy was initiated. One year later, he died during his sleep at a nursing home, probably due to an aspiration pneumonia. Post-mortem examination of the brain was consisted with MSA of the striatonigral degeneration type.

Hypohidrosis or anhidrosis due to loss of sweating function is a known, but not often described phenomenon of MSA [2-5]. Hyperthermia and subsequent loss of consciousness due to anhidrosis has been described occasionally, but in this case the patient was suffering from a concurrent infection [6]. Our patient showed no signs of underlying infection, which suggests that the summer heat - in combination with anhidrosis - was the cause of his hyperthermia. Although we did not perform a formal sweating test, both the patient's history and his physical examination were consistent with this conclusion. A malignant neuroleptic-like syndrome or acute akinesia as a possible explanation for his condition, which has been recently described by others [7], seems unlikely, since there was no marked rigidity. Furthermore, the patient was not receiving any levodopa therapy, dopamine agonists or neuroleptic drugs at the time of this event or in the previous days.

Body temperature increases when the rate of heat production exceeds the rate of heat dissipation. Hyperthermia occurs when thermoregulatory mechanisms are overwhelmed by excessive metabolic production of heat, excessive environmental heat, or impaired heat dissipation. In hyperthermic states, the hypothalamic set-point is normal but peripheral mechanisms are unable to maintain a body temperature that matches the set-point [8]. In our patient, hyperthermia was caused by a combination of excessive environmental heat and impaired heat dissipation due to hypohidrosis. Hypohidrosis in MSA is probably caused by both post- and preganglionic sudomotor failure [5], and a decreased potentiation of calcitonin gene related peptide (CGRP)-induced cholinergic sweat production [9]. It is possible that levodopa can further aggravate autonomic problems [10], but anhidrosis or heat stroke have not been described as adverse effects of levodopa.

Physicians should instruct patients with MSA and clear autonomic signs, in particular when this includes of hypo- or anhidrosis, to avoid extreme heat, and especially when suffering from an infection. When present, this hyperthermia must be rapidly treated by cooling the body with external cooling pads, and antibiotic therapy when necessary.

\section{References}

1. Gilman S, Low PA, Quinn N, et al. (1999) Consensus statement on the diagnosis of multiple system atrophy. J Neurol Sci 163:94-98
2. Shy GM, Drager GA (1960) A neurological syndrome associated with orthostatic hypotension. Arch Neurol 2:511-527

3. Cohen J, Low P, Fealey R, Sheps S, Jiang NS (1987) Somatic and autonomic function in progressive autonomic failure and multiple system atrophy. Ann Neurol 22:692-699

4. De Marinis M, Argenta G, Mele D, Carbone A, Baffigo G, Agnoli A (1993) Evaluation of vesico-urethral and sweating function in disorders presenting with parkinsonism. Clin Auton Res 3:125-130

5. Kihara M, Sugenoya J, Takahashi A (1991) The assessment of sudomotor dysfunction in multiple system atrophy. Clin Auton Res 1:297-302

6. Yamamoto K, Morita S, Ikeda S, Yanagisawa N (1993) Hyperthermia in a Shy-Drager syndrome patient pathophysiological effects of body temperature and L-DOPS on orthostatic hypotension. Rinsho Shinkeigaku 33:68-73

7. Onofrj M, Thomas A (2005) Acute akinesia in Parkinson disease. Neurology 64:1162-1169

8. Simon HB (1993) Hyperthermia. N Engl J Med 329:483-487

9. Kumazawa K, Sobue G, Mitsuma T, Sugenoya J, Ogawa T (1997) Impairment of calcitonin gene-related peptide-induced potentiation of cholinergic sweat secretion in patients with multiple system atrophy. Clin Auton Res 7:77-80

10. van Dijk JG, Haan J, Zwinderman K, Kremer B, van Hilten BJ, Roos RA (1993) Autonomic nervous system dysfunction in Parkinson's disease: relationships with age, medication, duration, and severity. J Neurol Neurosurg Psychiatry 56:1090-1095 